\title{
Archivos de Cardiología de México

\section{Determinación molecular de marcadores genéticos en síndromes coronarios agudos y su relación con eventos cardiovasculares adversos}

\author{
Luis Miguel Canseco-Ávila ${ }^{a}$, Carlos Jerjes-Sánchez ${ }^{b, *}$, Rocío Ortiz-López $^{\mathrm{c}}$ \\ y Augusto Rojas-Martínez ${ }^{\mathrm{c}}$
}

\author{
a Enseñanza e Investigación, Hospital Regional de Alta Especialidad Ciudad Salud, Tapachula, Chiapas, México \\ b Investigación Clínica, Instituto de Cardiología y Medicina Vascular TEC Salud del Sistema Tecnológico de Monterrey y Unidad de \\ Investigación Clínica en Medicina SC, Monterrey, NL, México \\ c Departamento de Bioquímica, Facultad de Medicina, Universidad Autónoma de Nuevo León, Monterrey, NL, México
}

Recibido el 28 de junio de 2011; aceptado el 14 de enero de 2013

\author{
PALABRAS CLAVE \\ Síndromes coronarios \\ agudos; \\ Cardiopatía \\ isquémica; \\ Polimorfismos; \\ México
}

\begin{abstract}
Resumen
Introducción: Algunos genes relacionados con proteínas que participan en algunas rutas metabólicas podrían tener un papel en el desarrollo de los síndromes coronarios agudos.

Objetivo: Correlación entre polimorfismos y su relación con eventos adversos en síndromes coronarios agudos.

Método: Prospectivo, seguimiento hospitalario y a un año. Inclusión: síndromes coronarios agudos con desnivel o elevación del ST secundario a aterotrombosis, estabilidad clínica. En todos, reacción de cadena de polimerasa y polimorfismos de la longitud de los fragmentos de restricción. Al estandarizar reacciones de cadena y genotipificación se realizó análisis preliminar de distribución de genotipos para cada polimorfismo y en ninguno se observaron desviaciones en la ley de equilibrio de Hardy-Weinberg $(p>0,05)$.

Resultados: De 2003 a 2005 se ingresaron 150 sujetos. Se analizaron 14 polimorfismos en 9 genes (fibrinógeno, factor v, VII, II, XIII, activador e inhibidor del plasminógeno-1 y proteína $\mathrm{C}$ reactiva). En síndromes coronarios agudos, un fibrinógeno $>450 \mathrm{mg} / \mathrm{dL}$ y leucocitos $>8,500$ cél $/ \mathrm{mm}^{3}$ fueron marcadores de mal pronóstico a un año. Los análisis de regresión identificaron al -148 CT/TT del fibrinógeno y al -717 AG/GG de la proteína $\mathrm{C}$ reactiva como marcadores de isquemia recurrente y al $1691 \mathrm{GA}+\mathrm{AA}$ para reinfarto.

Conclusión: En pacientes con síndromes coronarios agudos se demostró una relación entre polimorfismos relacionados con hemostasia e inflamación con eventos adversos, por lo que podrían considerarse marcadores de enfermedad coronaria. Se requiere una muestra mayor para confirmar estos resultados.

(C) 2011 Instituto Nacional de Cardiología Ignacio Chávez. Publicado por Masson Doyma México S.A. Todos los derechos reservados.
\end{abstract}

\footnotetext{
* Autor para correspondencia: San Patricio 112, Real San Agustín, CP 66278. San Pedro Garza García, Monterrey, NL, México. Teléfono: +0181 88880000 .

Correo electrónico: jerjes@prodigy.net.mx (C. Jerjes-Sánchez).
} 


\section{KEYWORDS}

Acute coronary syndromes;

Ischemic heart

disease;

Polymorphisms;

Mexico
Determination of molecular genetic markers in acute coronary syndromes and their relationship to cardiovascular adverse events

\begin{abstract}
Introduction: The genes coding for proteins due to their activity in several metabolic pathways could be related with the onset of acute coronary syndromes.

Objective: Relationship among polymorphisms and adverse events in.

Methods: Prospective. In - hospital, one - year follow-up. Inclusion Acute coronary syndromes with ST elevation or depression secondary to atherothrombosis, clinical stability. In all, polymerase chain reaction and length polymorphism of restriction fragments. By standardizing chain reactions and genotyping, a preliminary analysis of distribution of genotypes was performed for each polymorphism and no deviations were observed in the law of Hardy-Weinberg equilibrium $(P>.05)$.

Results: From 2003 to 2005, 150 subjects were enrolled. We analyzed 14 polymorphisms in 9 genes (fibrinogen, factor v, VII, II, XIII, plasminogen activator and inhibitor-1, C-reactive protein). In acute coronary syndromes, fibrinogen $>450 \mathrm{mg} / \mathrm{dL}$ and white blood count $8500 \mathrm{cells} / \mathrm{mm}^{3}$ were markers of poor prognosis to one year. Regression analysis identified the $-148 \mathrm{CT} / \mathrm{TT}$ and fibrinogen $-717 \mathrm{AG} / \mathrm{GG}$ of C-reactive protein as a marker of recurrent ischemia and reinfarction 1691GA + AA.

Conclusion: We are showing a relationship among polymorphisms involved in inflammation and hemostasis with adverse events in the acute phase and follow-up in acute coronary syndromes patients that could be considered as markers of ischemic heart disease. Larger sample is needed to confirm these results.

(C) 2011 Instituto Nacional de Cardiología Ignacio Chávez. Published by Masson Doyma México S.A. All rights reserved.
\end{abstract}

\section{Introducción}

Los síndromes coronarios agudos (SCA) son un término que da identidad a una constelación de síntomas secundarios a isquemia inducida por trombosis aguda $(>85 \%)^{1,2}$. Su espectro fisiopatogénico (aterotrombosis) incluye una compleja interacción entre medio ambiente, inflamación, disfunción endotelial y mensajes moleculares. En un extremo del espectro, la trombosis depende de una interacción entre factores de inflamación y hemostáticos que pueden contribuir a la extensión y persistencia del trombo, al fracaso terapéutico y a eventos cardiovasculares adversos mayores $(E C A M)^{3}$. En el otro extremo, los polimorfismos por su actividad en algunas rutas metabólicas se relacionan con el inicio de la enfermedad y por su participación en inflamación, trombosis y trombo-resistencia podrían tener importantes implicaciones en la evolución. Por esta evidencia y por su importancia en prevención primaria y secundaria realizamos un estudio prospectivo para determinar la relación entre polimorfismos y ECAM.

\section{Métodos}

Estudio prospectivo de cohortes con seguimiento hospitalario y a un año. Objetivo principal: correlación entre polimorfismos (marcadores de enfermedad coronaria [EC]) inestable y estable) y su relación con ECAM en SCA. Objetivos secundarios: a) frecuencia en 3 cohortes, SCA, EC estable (ECE) y sujetos normales; b) identificar en fase aguda parámetros bioquímicos y hematológicos y compararlos con controles; c) determinar y correlacionar polimorfismos con los niveles plasmáticos de factores bioquímicos y hematológicos, y ECAM; y d) seguimiento en el grupo de SCA para ECAM adversos. Inclusión: a) edad entre 35 y 75 años; b) SCA con desnivel o elevación del ST, con o sin necrosis secundario a aterotrombosis demostrado angiográficamente; c) estabilidad clínica. Exclusión: a) > 75 años; b) SCA secundario a estrés, anemia, etcétera; c) Killip clase > I; d) SCA en los 3 últimos meses; e) fracción de expulsión < $35 \%$; f) enfermedad hematológica, hepática o neoplásica; g) actividad inflamatoria aguda o crónica; y h) consumo excesivo de alcohol.

\section{Controles}

Enfermedad coronaria estable: pacientes con diagnóstico previo de cardiopatía isquémica por aterosclerosis coronaria demostrada angiográficamente, sin dolor con perfil isquémico en reposo o ejercicio y sin expresión electrocardiográfica de isquemia aguda en los últimos 6 meses. Sujetos sanos: sin enfermedad cardiovascular excluida por historia clínica, examen físico, signos vitales, laboratorio, radiografía de tórax, electrocardiograma y ecocardiograma. Ambos grupos se eligieron por edad y sexo con relación al grupo en estudio.

El protocolo y el consentimiento informado fueron aprobados por los Comités de Investigación y Ética del Hospital de Cardiología No 34 y Hospital Universitario Dr. José E. González de la Universidad Autónoma de Nuevo León. Todos los pacientes leyeron y firmaron el consentimiento informado.

\section{Selección de genes}

Por la complejidad de los polimorfismos la selección genética (rutas metabólicas) se realizó utilizando datos 
Tabla 1 Genes y polimorfismos estudiados

\begin{tabular}{lll}
\hline $\begin{array}{l}\text { Ubicación } \\
\text { (cromosoma) }\end{array}$ & Gen & Polimorfismo \\
\hline 1 & Factor v & Arg506Gln \\
& $\begin{array}{l}\text { Proteína C } \\
\text { reactiva }\end{array}$ & $\mathrm{y}-717 \mathrm{~A} / \mathrm{G}$ \\
& Fibrinógeno & $\mathrm{Bcl} 1, \mathrm{G}-445 \mathrm{~A}$ \\
4 & & $\mathrm{C} / \mathrm{T}-148, \mathrm{Taq}$ I, \\
& & $\mathrm{T} / \mathrm{G}-1689$ \\
& Factor xIII & V34L \\
6 & IATP-1 & -675 4G/5G \\
8 & ATP & Alu II/D \\
11 & Factor ॥ & G20210A \\
13 & Factor vII & Arg353Glu \\
& Glucoproteína IIIa & T1565C \\
& & (PLA1/PLA2) \\
\hline
\end{tabular}

ATP: activador tisular del plasminógeno; IATP-1: inhibidor del activador tisular del plasminógeno 1 .

previamente publicados en donde demostramos asociación entre marcadores bioquímicos (fibrinógeno, plasminógeno, antitrombina III, proteína C activada, proteína S, inhibidor de la proteína $C, a^{2}$-antiplasmina y proteína $C$ reactiva) con $\operatorname{ECAM}^{3,4}$ (tabla 1).

\section{Técnica de extracción del ADN}

Se extrajo mediante la técnica TSNT (Tritón x-100. SDS. $\mathrm{NaCl}$ y Tris-EDTA) previamente descrita ${ }^{5}$.

\section{Reacciones de cadena de polimerasa de los genes}

Ciclos con etapas de desnaturalización, alineamiento y extensión. En presencia de la TaqDNA polimerasa, $\mathrm{MgCl}_{2}$ y dNTPs, los oligonucleótidos se tornan híbridos con la secuencia blanco específica ${ }^{5}$. En la detección de los genotipos se utilizaron oligonucleótidos específicos.

\section{Polimorfismos de la longitud de los fragmentos de restricción}

Se detectaron fragmentos de ADN posterior a su digestión por enzimas de restricción. Los productos amplificados fueron digeridos de acuerdo con las condiciones de reacción establecidas. Para asegurar la calidad de la validación se realizó un tamizaje al azar en el $10 \%$ de las muestras y se tamizaron aquellas con patrón homocigoto para alelos mutantes $^{6}$.

\section{Definición de eventos cardiovasculares adversos mayores}

La definición de isquemia recurrente, reinfarto, choque cardiogénico y defunción cardiovascular ha sido previamente publicada ${ }^{3}$. En resumen, para isquemia, nuevo dolor torácico con perfil isquémico y cambios dinámicos del ST (elevación o desnivel) en 2 derivaciones contiguas; para reinfarto, necrosis por electrocardiograma y biomarcadores; y para oclusión de la arteria relacionada, demostración angiográfica. Para choque cardiogénico se utilizaron variables clínicas, uso de vasopresores y datos hemodinámicos. Se consideró defunción cardiovascular toda mortalidad atribuida al evento índice de inestabilidad coronaria ${ }^{3}$.

\section{Seguimiento}

Llamadas telefónicas y/o visita de consultorio a los 3, 6, 9 y 12 meses.

\section{Análisis estadístico}

Se evaluó la diferencia entre medias de grupos y variables continuas mediante $\mathrm{t}$ de Student de 2 colas; los resultados se verificaron con la prueba no paramétrica de la suma del rango de Wilcoxon. Se recogieron las variables discretas mediante chi-cuadrado con corrección de Yates o prueba exacta de Fisher. Se usaron razón de momios (RM) y riesgo relativo con intervalos de confianza (IC) del 95\% (IC 95\%) para determinar probabilidad, riesgo de enfermedad y ECAM. Se utilizaron modelos univariados de regresión logística y multivariada para identificar asociaciones entre variables; y Kaplan-Meier para ECAM (mortalidad, isquemia recurrente, reinfarto, choque cardiogénico y mortalidad) y supervivencia. Significación estadística: $p<0,05$. Los datos se miden en porcentajes, medias, desviaciones estándar, IC y RM. Se utilizó el paquete estadístico GBSTAT versión 10.0 of Dynamic Microsystems, Inc., Copyright 2004.

\section{Resultados}

De septiembre del 2003 a agosto del 2005, 150 sujetos ingresaron al estudio. Las características demográficas de los 3 grupos se observan en la tabla 2 . El promedio de edad fue alrededor de los 60 años con predominio de hombres. El tabaquismo fue una variable dominante en los pacientes con ECE y SCA en relación con los controles. Aunque estos se encontraban libres de enfermedad cardiovascular se observó tabaquismo, obesidad y sedentarismo en proporción similar a los otros grupos. La presencia de SCA con y sin elevación del ST fue similar en EC inestable. Angiográficamente en todos se demostró aterotrombosis. No se tuvo acceso a ultrasonido intracoronario.

\section{Estandarización de la detección de polimorfismos}

Se analizaron 14 polimorfismos en 9 genes. Fibrinógeno: 5 polimorfismos, 4 en el gen de la cadena $\beta(-455 \mathrm{G} / \mathrm{A}$, $-148 \mathrm{C} / \mathrm{T},+1689 \mathrm{~T} / \mathrm{Gy} \mathrm{Bcl}-1)$ y uno en la cadena $\alpha$ (Taq ।). Factor v: G1691A. Factor vII: R353Q. Factor I: G20210A. Factor XIII: V34L. Inhibidor del activador del plasminógeno1 (IAP-1): 4G/5G. Para el gen del activador tisular del plasminógeno (ATP): Alul/D. PLA1/PLA2: glucoproteína Illa, PLA1/PLA2. Proteína C reactiva: G1059C y el $-717 A / G$.

\section{Frecuencias de polimorfismos en las 3 cohortes}

Un análisis preliminar de distribución de genotipos para cada polimorfismo no demostró desviaciones en la ley de 
Tabla 2 Características demográficas

\begin{tabular}{|c|c|c|c|c|}
\hline Característica & Controles $\mathrm{n}=50(\%)$ & ECE $n=50(\%)$ & $\mathrm{SCA} n=50(\%)$ & $\mathrm{p}$ \\
\hline Edad (años, \pm DE) & $59.5 \pm 7.4$ & $61.9 \pm 8.7$ & $61.7 \pm 8.2$ & NS \\
\hline Mujeres & $18(36)$ & $17(34)$ & 17 (34) & \\
\hline Hombres & $32(64)$ & $33(66)$ & $33(66)$ & NS \\
\hline Tabaquismo & $14(28)$ & $23(46)$ & $27(54)$ & 0.0007 \\
\hline Obesidad & $16(32)$ & $12(24)$ & $17(34)$ & NS \\
\hline Diabetes & 0 & $22(52)$ & $25(50)$ & NS \\
\hline Hipertensión & 0 & $25(60)$ & $28(56)$ & NS \\
\hline Dislipidemia & 0 & 7 (17) & $13(26)$ & NS \\
\hline Vida sedentaria & $27(54)$ & $27(54)$ & $32(64)$ & NS \\
\hline IMC & $27 \pm 4.9$ & $29 \pm 7.5$ & $29 \pm 7.5$ & NS \\
\hline SCA con elevación ST & - & - & $28(58)$ & \\
\hline SCA sin elevación ST & - & - & $22(44)$ & \\
\hline
\end{tabular}

ECE: enfermedad coronaria estable; IMC: índice de masa corporal; NS: no significativo; SCA: síndromes coronarios agudos.

equilibrio de Hardy-Weinberg ( $p>0,05)$. De los 4 estudiados, solo los localizados en el promotor del gen del fibrinógeno $(-455 \mathrm{G} / \mathrm{A}$ y $-148 \mathrm{C} / \mathrm{T})$ y Taq I (gen fibrinógeno $\alpha$ ) tuvieron diferencia estadísticamente significativa al comparar SCA, ECE y controles (tabla 3). La frecuencia de los genotipos $-455 \mathrm{G} / \mathrm{A}$ y $-48 \mathrm{C} / \mathrm{T}$ fue más significativa en los grupos con ECE o inestable $(p=0,001$ y $p<0.0001)$. Sin embargo, al analizar los alelos solo la variante $-148 \mathrm{~T}$ tuvo significación estadística $(p=0,004)$. El resto no mostraron valores significativos para una condición clínica particular.

Al analizar la diferencia de frecuencias entre grupos solo los polimorfismos R353Q y V34L demostraron significación $(p=0,03$ y $p<0.0001)$. Esta misma comparación con alelos sostuvo al V34L con significación $(p<0.0001)$. Al analizar la diferencia de frecuencia entre grupos, solo los polimorfismo Alu $D /$ / y $-717 \mathrm{~A} / \mathrm{G}$ tuvieron significación estadística $(p=0,008$ y 0.0002$)$ y al compararlos con los alelos solo el Alu $D /$ / permaneció estadísticamente significativo.

La determinación de las cargas genéticas de un alelo en particular entre los genotipos estudiados demostró incremento para EC. También se observó cómo la frecuencia de la carga del alelo -455A tuvo significado (RM 2.66, IC 95\% 1.1 6.3, $p=0,03$ ) para las 2 condiciones patológicas. La carga del alelo $-148 \mathrm{~T}$ fue significativo para el grupo con ECE (RM 2.25 , IC 95\% 1.0-4.9, $p=0,04$ ) y para SCA (RM 2.93, IC 95\% 1.3-6.4, $p=0,01)$. El alelo $-717 \mathrm{G}$ solo tuvo significación en sanos y SCA (RM 9.33, $p=0,03$ ).

\section{Variables bioquímicas}

Las variables bioquímicas con mayor significado estadístico entre sanos y ECE fueron: fibrinógeno $(371.2+97.4$ vs. $414.6+94.3 \mathrm{mg} / \mathrm{dL}, \mathrm{p} 0,03)$, leucocitos $(6.212+1.543$ vs. $7.181+2.113 \mathrm{cél} / \mathrm{mm}^{3}$, p 0,01$)$, neutrófilos $(3.863 \pm 1.344$ vs. $\left.4.635 \pm 1.623 \mathrm{cél} / \mathrm{mm}^{3}, \mathrm{p} 0,01\right)$, creatinina $(0,87 \pm 0,2$ vs. $1.0+0,34 \mathrm{mg} / \mathrm{dL}, \mathrm{p} \mathrm{0,02)}$.

Actividad de factores: factor ॥ $(103.1+26.4$ vs. $80.9 \pm 21.5 \%, p<0,001)$, factor $\vee(97.2 \pm 33.1$ vs. $72 \pm 26.7 \%$, p 0,001$)$ y factor VII $(122.9 \pm 32.0$ vs. $93.8 \pm 33.4 \%$, $\mathrm{p}<0,001)$.

Al comparar controles con SCA sostuvieron su significado estadístico: fibrinógeno $\quad(371.2 \pm 97.4$ vs.
$442.5+153.2 .3 \mathrm{mg} / \mathrm{dL}, \mathrm{p} \mathrm{0,007);} \mathrm{leucocitos}(6.212+1.543$ vs. $\quad 8.982+3.244$ cél $\left./ \mathrm{mm}^{3}, \quad \mathrm{p}<0,001\right)$; neutrófilos $\left(3.863 \pm 1.344\right.$ vs. $6.727 \pm 2.932$ cél $\left./ \mathrm{mm}^{3}, \quad \mathrm{p}<0.0001\right)$; creatinina $(0,87+0,2$ vs. $1 \cdot 1+0,63 \mathrm{mg} / \mathrm{dL}, \mathrm{p} 0,02)$ y la actividad del factor ॥ $(103.1+26.4$ vs. $83.8 \pm 24.8 \%$, p 0.0003).

Al concertar ECE con SCA mantuvieron significación: leucocitos $\left(71.781 \pm 2.113\right.$ vs. $8.982 \pm 3.244$ cél $\left./ \mathrm{mm}^{3}, \mathrm{p} 0,001\right)$ y neutrófilos $\left(4.635 \pm 1.623\right.$ vs. $6.727 \pm 2.932$ cél $/ \mathrm{mm}^{3}$, $p<0.0001)$ y los factores $v(72 \pm 26.7$ vs. $84.7 \pm 29.9 \%)$ y VII $(93.8 \pm 33.4$ vs $140.1 \pm 97.5 \%)$. Aunque el fibrinógeno no demostró ninguna diferencia $(414.6 \pm 94.3$ vs. $442.4 \pm 153.2 \mathrm{mg} / \mathrm{dL}, \mathrm{p} 0,30$ ), las cifras observadas (> $350 \mathrm{mg} / \overline{\mathrm{dL}}$ ) han demostrado ser una variable independiente de riesgo cardiovascular ${ }^{4}$.

\section{Relación entre polimorfismos y niveles plasmáticos del fibrinógeno}

El polimorfismo Bcl-1 (B1B2) se observó en el grupo con niveles entre 250 y $350 \mathrm{mg} / \mathrm{dL}$ en el $14 \%$; con un nivel entre 350 y $450 \mathrm{mg} / \mathrm{dL}$ en el $24 \%$ y > $450 \mathrm{mg} / \mathrm{dL}$ en el $34 \%$ (p 0,004). El polimorfismo $-455 \mathrm{Fg}(\mathrm{G} / \mathrm{A})$ se identificó en el 14,36 y $50 \%$ respectivamente $(\mathrm{p}<0,001)$. $\mathrm{El}-148 \mathrm{Fg}(\mathrm{C} / \mathrm{T})$ en el $46,49 \mathrm{y}$ $43 \%(0,001)$. El Tap1Fg (T1/T2) en el 33, 33 y 34\% $(0,06)$. El polimorfismo $+1689 \mathrm{Fg}$ en un 26,38 y $52 \%(0.0015)$. Los modelos de regresión múltiple que incluyeron variables históricas para ECAM (tabaquismo, edad, sexo, diabetes, dislipidemia, etcétera) tuvieron la mayor correlación cuando se asociaron los polimorfismos del fibrinógeno -455 y $-148(r=0,3$, $p=0,008$ ).

En la figura 1 se observa en las 3 cohortes la distribución de los 2 polimorfismos del fibrinógeno con mayor impacto estadístico. El polimorfismo del factor vIII se puede analizar en los 3 grupos en la figura 2. La figura 3 muestra la distribución genotípica del polimorfismo de la PCR $(-717 \mathrm{~A} / \mathrm{G})$ en las 3 cohortes de pacientes.

\section{Eventos cardiovasculares adversos mayores}

La mayor incidencia de isquemia recurrente, reinfarto, choque y mortalidad se observó en la fase aguda. Isquemia 
Tabla 3 Genotipos del fibrinógeno en los grupos de estudio

\begin{tabular}{|c|c|c|c|c|}
\hline Genotipos & Controles $\mathrm{n}=50(\%)$ & ECE $n=50(\%)$ & $\mathrm{SCA} n=50(\%)$ & $\mathrm{p}$ \\
\hline \multicolumn{5}{|c|}{ Fibrinógeno } \\
\hline \multicolumn{5}{|l|}{-455} \\
\hline $\mathrm{G} / \mathrm{G}$ & $40(80)$ & $30(60)$ & $30(60)$ & \\
\hline G/A & $9(18)$ & $20(40)$ & $20(40)$ & 0.0013 \\
\hline $\mathrm{A} / \mathrm{A}$ & $1(2)$ & $0(0)$ & $0(0)$ & \\
\hline \multicolumn{5}{|l|}{-148} \\
\hline $\mathrm{C} / \mathrm{C}$ & $29(58)$ & $19(38)$ & $16(32)$ & \\
\hline $\mathrm{C} / \mathrm{T}$ & $20(40)$ & $26(52)$ & $24(48)$ & $<0.0001$ \\
\hline $\mathrm{T} / \mathrm{T}$ & $1(2)$ & $5(10)$ & $10(20)$ & \\
\hline \multicolumn{5}{|l|}{+1689} \\
\hline $\mathrm{T} / \mathrm{T}$ & $32(64)$ & $31(62)$ & $30(60)$ & \\
\hline $\mathrm{G} / \mathrm{T}$ & $17(34)$ & $19(38)$ & $20(40)$ & NS \\
\hline G/G & $1(2)$ & $0(0)$ & $0(0)$ & \\
\hline \multicolumn{5}{|l|}{ Taq । } \\
\hline $\mathrm{T} 1 / \mathrm{T} 1$ & $29(58)$ & $38(76)$ & $30(60)$ & \\
\hline $\mathrm{T} 1 / \mathrm{T} 2$ & $19(38)$ & $12(24)$ & $18(36)$ & 0,03 \\
\hline $\mathrm{T} 2 / \mathrm{T} 2$ & $2(4)$ & 0() & $2(4)$ & \\
\hline \multicolumn{5}{|l|}{$B c l-1$} \\
\hline B1/B1 & $39(78)$ & $38(76)$ & $38(76)$ & \\
\hline B1/B2 & $11(22)$ & $12(24)$ & $12(24)$ & NS \\
\hline B2/B2 & $0(0)$ & $0(0)$ & $0(0)$ & \\
\hline \multicolumn{5}{|l|}{ Alelos } \\
\hline \multicolumn{5}{|l|}{-455} \\
\hline G & 0,89 & 0,80 & 0,80 & NS \\
\hline A & 0,11 & 0,20 & 0,20 & \\
\hline \multicolumn{5}{|l|}{-148} \\
\hline C & 0,78 & 0,64 & 0,56 & 0,004 \\
\hline $\mathrm{T}$ & 0,22 & 0,36 & 0,44 & NS \\
\hline \multicolumn{5}{|l|}{+1689} \\
\hline $\mathrm{T}$ & 0,81 & 0,81 & 0,80 & NS \\
\hline G & 0,19 & 0,19 & 0,20 & NS \\
\hline \multicolumn{5}{|l|}{ Taq । } \\
\hline $\mathrm{T} 1$ & 0,77 & 0,88 & 0,78 & \\
\hline $\mathrm{T} 2$ & 0,23 & 0,12 & 0,22 & \\
\hline \multicolumn{5}{|l|}{$B C l$} \\
\hline B1 & 0,89 & 0,88 & 0,88 & \\
\hline B2 & 0,11 & 0,12 & 0,12 & \\
\hline
\end{tabular}

ECE: enfermedad coronaria estable; NS: no significativo; SCA: síndromes coronarios agudos.

recurrente: hospitalaria 10\%, 3 meses $8 \%, 6$ meses $5 \%, 9$ meses $6 \%$ y 12 meses 5\%. Reinfarto: hospitalario 2\%, 3 meses $3 \%$ y 9 meses 1\%. Choque: hospitalario 3\%. Mortalidad: hospitalaria $3 \%, 3$ meses de seguimiento $1 \%$.

\section{Correlación entre variables bioquímicas, hematológicas y polimorfismos}

Seguimiento a un año. En el grupo con SCA los niveles plasmáticos de fibrinógeno $>450 \mathrm{mg} / \mathrm{dL}(p=0,03)$ y leucocitos $>8.500 \mathrm{cél} / \mathrm{mm}^{3}(\mathrm{p}=0,05)$, fueron marcadores de mal pronóstico. Se identificó la carga genética -148 CT/TT del fibrinógeno (RM 2.0, p0,04) y el -717 AG/GG de la PCR (RM 2.0, p 0,04) como marcadores de isquemia recurrente. Para reinfarto se asoció el polimorfismo 1691GA + AA (RM 2.4, p 0,01). Los neutrófilos fueron una variable independiente para choque cardiogénico (RM 2.0, p 0,04) y la disfunción del ventrículo derecho para mortalidad (RM 1.9, p 0,05).

El modelo de regresión multivariado para isquemia recurrente $(p=0,002)$ fue dominantemente genético e incluyó angiografía coronaria (RM 2.12, p 0,03), -148 CT + TT (RM 2.03, p 0,04), PLA1/PLA2 + PLA2/PLA2 (RM 2.28, p 0,02). A un año de seguimiento, el modelo de regresión multivariado con mayor poder estadístico para mortalidad incluyó variables históricas, marcadores indirectos de disfunción endotelial por inflamación y el genotipo Alu I// (p 0,001). Las variables con significación estadística fueron fracción de expulsión (RM 1.96, p 0,04) y el Alu ı/। (RM 2.16, p 0,03). El modelo de regresión multivariado para ECAM a un año de seguimiento $(p=0,04)$ incluyó variables clínicas como hipertensión, angiografía coronaria, lesiones críticas y 2 cargas genotípicas en donde el polimorfismo -148 CT/TT del fibrinógeno fue el más significativo (RM 1.91, p 0,05). 


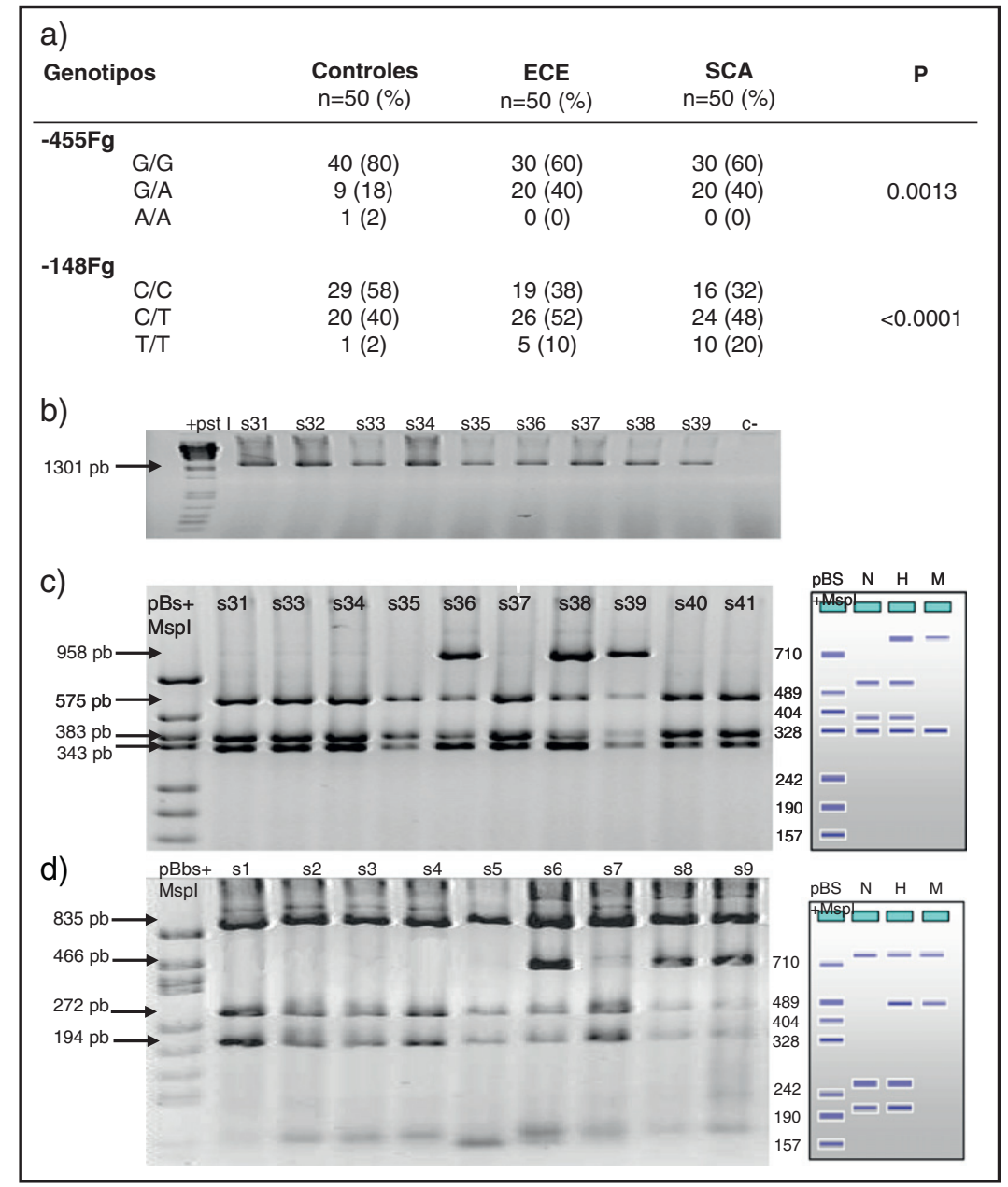

Figura 1 En la parte izquierda se encuentra el esquema del gel de agarosa del polimorfismo -455 G/A con el marcador PM y en el carril $\mathrm{N}$ se encuentra representado el homocigoto $\mathrm{G} / \mathrm{G}$, en el carril $\mathrm{H}$ el heterocigoto $\mathrm{G} / \mathrm{A}$ y en el $\mathrm{M}$ el homocigoto AA. A la derecha un gel de agarosa del polimorfismo $-148 \mathrm{C} / \mathrm{T}$ y como las muestras s31, s33, s34, s35, s37, s40 y s41 son homocigotas G/G y las muestras s36, s38 y s39 son heterocigotas G/A para el polimorfismo, -455 .

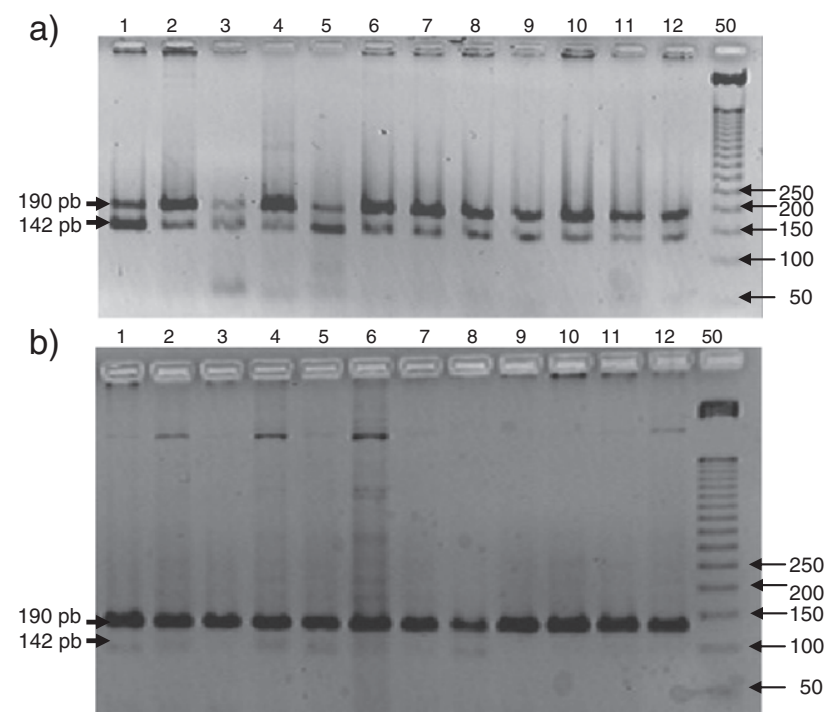

Figura 2 Se puede analizar el polimorfismo del factor VIII en los 3 grupos.

\section{Seguimiento}

Se realizó seguimiento hospitalario a los 3, 6, 9 y 12 meses a través de visita médica y/o contacto telefónico. Esto se logró en el $97 \%$ de los casos. En los primeros 9 meses se perdió el contacto con 2 pacientes y a los 12 meses con otros 2.

\section{Discusión}

Este estudio tiene 3 importantes hallazgos. Primero, la distribución genotípica de los genes sugiere una relación entre polimorfismos del fibrinógeno y de la hemostasia como marcadores de ECAM y de EC. Segundo, propone que la hiperfibrinogenemia que demostramos en diferentes modelos $2,3,7$ parece tener una predisposición genética ${ }^{2,3}$ más que ambiental ${ }^{4}$. Tercero, en nuestro medio $y$ en diferentes estadios de $\mathrm{EC}^{4}$, se confirma el valor predictivo de leucocitos y fibrinógeno ${ }^{2,3,7,8}$.

En México la necesidad de estudios genéticos surge por las crecientes tasas de morbimortalidad e incapacidad 


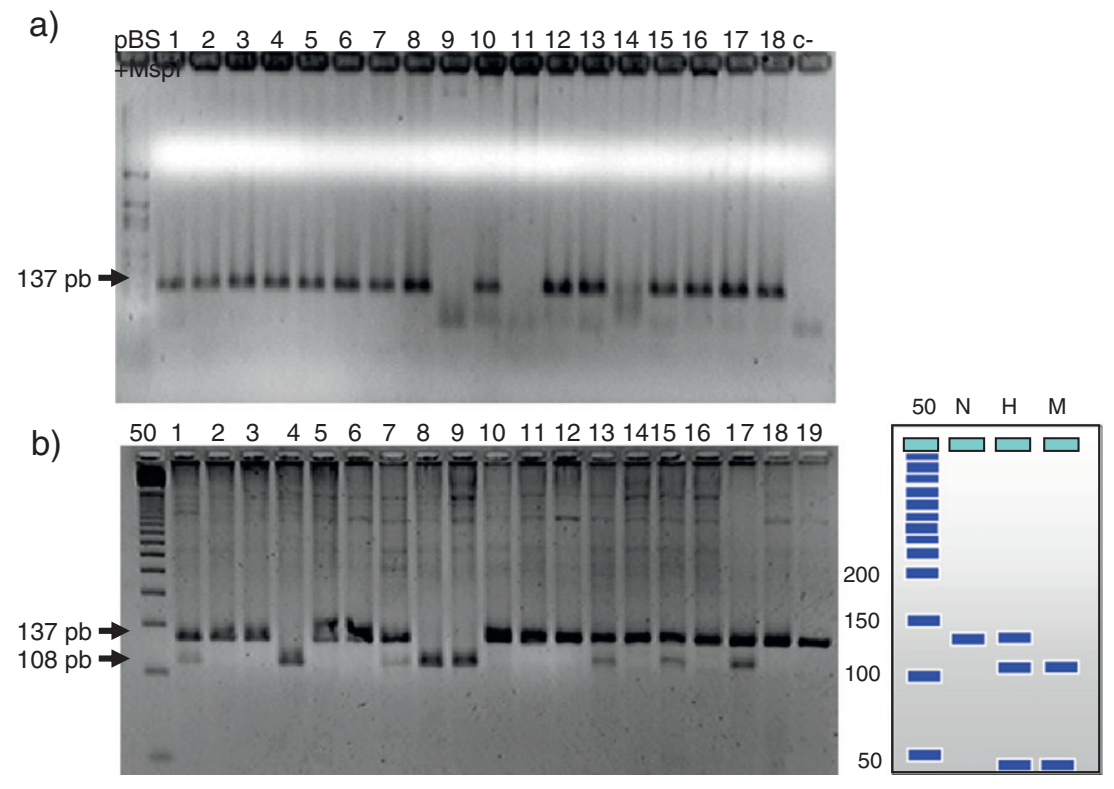

Figura 3 Se observa la distribución genotípica del polimorfismo de la PCR $(-717 \mathrm{~A} / \mathrm{G})$ en las 3 cohortes de pacientes.

por enfermedad vascular ${ }^{9-12}$, la variabilidad genética demostrada en diferentes grupos étnicos, la necesidad de identificar un perfil de susceptibilidad génica y porque en el futuro este puede ser el camino para identificar riesgo vascular, respuesta o nuevos objetivos terapéuticos y establecer efectivas estrategias de prevención primaria.

\section{Enfermedad coronaria y genética}

En la década pasada la aterosclerosis dejó de ser un padecimiento por depósito de colesterol y hoy se acepta como una enfermedad inflamatoria con una compleja interacción entre medio ambiente, disfunción endotelial y mensajes moleculares ${ }^{1,2}$. Además, las evidencias que demuestran que el endotelio va más allá de los vasa vasorum ${ }^{13}$, el concepto de remodelación vascular como expresión biológica de placas no estenóticas ${ }^{1,2}$, ruptura múltiple $e^{14,15}$ y la participación de factores de riesgo aterotrombóticos en la enfermedad tromboembólica venosa pulmonar ${ }^{16}$, le confieren una expresión de enfermedad sistémica, por lo que el concepto de «paciente vulnerable» debe extenderse al de «endotelio y sistema vascular vulnerable ${ }^{16}$.

A través de avances en biología molecular se han detectado numerosos polimorfismos y mutaciones que al establecer un sinergismo con factores ambientales podrían estar implicados no solo en la génesis de la aterosclerosis (inflamación) ${ }^{17}$ sino también en algunos mecanismos íntimamente relacionados (metabolismo de los lípidos, resistencia a insulina, etcétera) con inestabilidad de la placa (actividad y reclutamiento de células inflamatorias y trombosis) ${ }^{17}$. Todo esto confiere a la EC un carácter poligénico y multifactorial, y establece la susceptibilidad genética como un factor de riesgo tan importante como los factores tradicionales.

\section{Distribuciones genotípicas y polimorfismos}

La distribución de los genes, además de establecer un vínculo entre el conocimiento biomolecular y los SCA ${ }^{12}$, podría ser - hasta nuestro conocimiento- la primera evidencia reportada en el país. Una aportación importante fue demostrar la ausencia de polimorfismos de riesgo cardiovascular observados en poblaciones caucásicas (G20210A del factor II y G1691A del factor v) ${ }^{18-22}$.

Otro resultado importante fue identificar una relación entre polimorfismos de inflamación (fibrinógeno -148T) y coagulación (factores XIII V34L, ATP Alu ।/D y PLA2 del GIIla) con ECAM como expresión de un posible mecanismo multifactorial similar al observado con marcadores bioquímicos ${ }^{3}$. La misma expresión genética e impacto clínico se extendió en el seguimiento en donde polimorfismos de la misma clase (fibrinógeno $-148 \mathrm{Fg},-455 \mathrm{G} / \mathrm{A}$ y factor XIII V34L) también se relacionaron con EC.

Los polimorfismos en el promotor del fibrinógeno se vincularon con el fenotipo por una asociación independiente con los factores de riesgo históricos. Estos niveles de la proteína fueron superiores a otras evidencias ${ }^{23,24}$. En todo caso, las diferencias estadísticas observadas entre los grupos sugieren estratificaciones fenotípicas que pudieran relacionarse con un mayor riesgo cardiovascular.

$$
\text { Alelos }-455 \text { A y }-148 \mathrm{~T}
$$

Al comparar sus cargas genéticas se observó relación con EC, lo que sugiere que los polimorfismos localizados en el promotor de la cadena $\beta$ son responsables de regular los niveles de fibrinógeno asociados con enfermedad. El alelo $-455 \mathrm{~A}$ en diferentes grupos étnicos ${ }^{25-28}$ ha demostrado una relación inconsistente con cardiopatía isquémica ${ }^{29,30}$ y el -148 $T$ tiene una evidencia limitada como riesgo cardiovascular en poblaciones asiáticas y anglosajonas con infarto ${ }^{31}$ o enfermedad arterial periférica ${ }^{32}$. Por ello, esta evidencia podría ser la primera en establecer una relación entre estos alelos y EC en mexicanos.

En la fase aguda (alelo -455 A) y en el seguimiento (alelo -148 T) se observó una relación estrecha con valores anormales de fibrinógeno y ECAM, por lo que el alelo -148 
T podría ser un marcador de EC. La asociación de ambos sugiere la posibilidad de un desequilibrio de ligamiento entre estos polimorfismos, sin que esto pueda establecerse por completo $^{33,34}$. Su localización estratégica en la región promotora del gen del fibrinógeno $\beta$ cerca del elemento de respuesta de la interleucina 6 y C/EBP, y de los sitios HNF1 y HNF3 ${ }^{27,35-39}$, es esencial para una respuesta completa del promotor a esta interleucina responsable de la respuesta inflamatoria y de niveles elevados de reactantes de fase aguda (fibrinógeno y proteína $\mathrm{C}$ ). Su relación con ECAM podría atribuirse a una mayor respuesta del promotor del fibrinógeno a diferentes estímulos que generan un estado de disfunción endotelial agudo.

\section{Alelo V34L}

En este polimorfismo del factor xIII el cambio de aminoácido se encuentra muy cerca del anclaje de la trombina lo que podría ser muy importante en la activación de este factor. In vitro la 34 leucina parece formar trombos con mayor estabilidad a la fibrinólisis endógena ${ }^{40}$ sin embargo, otras evidencias sugieren que se asocia a trombo-resistencia e infarto ${ }^{41,42}$. Esto podría atribuirse a la interacción entre el factor XIII y la estructura de la fibrina con la actividad del fibrinógeno. Su alta concentración en muestras homocitogos para Leu se relaciona con trombos con mayor permeabilidad y una estructura más laxa con fibras densas que en aquellos formados por las muestras homocigotos Val. Por lo tanto el factor XIII en presencia de niveles de fibrinógeno relacionados con riesgo cardiovascular ${ }^{4}$ podría generar un mecanismo de trombo-resistencia a la fibrinólisis endógena y exógena con todas sus implicaciones clínicas.

\section{Alelo PLA2 del GPIIla}

Su importancia como marcador de isquemia recurrente se establece por su localización en el sitio de unión del fibrinógeno y la relación que parece existir entre inflamación y mecanismos de agregación plaquetaria ${ }^{43}$; esto sugiere que este alelo incrementa la actividad plaquetaria por una interacción con los niveles del fibrinógeno y mayor trombogénesis, y es una evidencia más de la interacción biológica entre plaquetas, leucocitos, trombosis e inflamación ${ }^{43}$.

\section{Alelo Alu I/ı}

Aunque evidencias previas no lo han correlacionado con el ATP ${ }^{44-46}$, este alelo fue un marcador de mortalidad lo que sugiere que una hipoactividad de la fibrinólisis asociada a una reducción de sus niveles plasmáticos puede ser un factor de riesgo para trombosis. La naturaleza de inserción de una repetición Alu en un intrón hace difícil que esta mutación afecte los niveles de esta proteína; sin embargo, es posible alterar la estabilidad del $\mathrm{mARN}^{47}$ considerando que las concentraciones del ATP dependen de su secreción y separación, así como del complejo que forma con el IAP- $1^{48}$.

Por otra parte, la determinación del ATP secretado por células endoteliales supera a la determinación de niveles circulantes ${ }^{49}$. Posterior a su expresión endotelial pierde rápidamente actividad por la separación mediada por receptores del hígado y la interacción del IAP-1. Su mayor actividad lítica sobre la fibrina se observa en el trombo formado ${ }^{50,51}$ y cuando su secreción en las células endoteliales es mayor que los niveles circulantes. Datos recientes apoyan la relación entre el polimorfismo Alu I/D y la secreción del ATP ${ }^{52,53}$ lo que sugiere que este alelo se asocia con un sistema fibrinolítico anormal y trombogénesis.

\section{Leucocitos y fibrinógeno}

Como hemos demostrado previamente ${ }^{3,7}$, ambos marcadores indirectos de disfunción endotelial e inflamación fueron marcadores de mal pronóstico. El mecanismo parece ser multifactorial. Los leucocitos se asocian a hipercoagulabilidad, fenómeno de no-reflujo, interleucinas y moléculas de adhesión celular-1. El fibrinógeno participa en la formación de trombina, agregación plaquetaria, modula la disfunción endotelial, promueve proliferación y migración de las células del músculo liso, interactúa con las uniones de plasmina y es una proteína mayor de fase aguda ${ }^{4}$. Los alelos del fibrinógeno demostrados sugieren una posible participación genética más que ambiental ${ }^{4}$. Si ambos se encuentran involucrados o no en la causalidad de la aterotrombosis y en la génesis de ECAM, está aún por determinarse. Sin embargo, mientras esta interrogante y otras esperan una respuesta, estos indicadores de inflamación emergen como promisorios marcadores adicionales de riesgo cardiovascular que podrían estratificar el riesgo en diferentes estadios de la EC.

\section{Implicaciones clínicas}

Los marcadores genéticos y bioquímicos demostrados son una evidencia más de la compleja interacción entre medio ambiente, inflamación, disfunción endotelial y mensajes moleculares. La evidencia actual establece al fibrinógeno ${ }^{4}$ como un factor fuerte, consistente e independiente o un indicador de riesgo, y podría ser el vínculo perdido entre la enfermedad cardiovascular y los factores clásicos de riesgo. Además, por su participación activa en mecanismos de resistencia o respuesta subóptima a heparina ${ }^{4}$, terapia fibrinolítica ${ }^{4}$ e inhibidores de los receptores de superficie plaquetaria $\mathrm{Ilb} / \mathrm{Illa}^{54}$, podría ser un importante predictor de respuesta terapéutica. Estos resultados podrían considerarse como un estudio piloto para generar un estudio representativo en población mexicana y mejorar su validez interna y externa.

\section{Limitaciones}

Muestra y población limitada, por el costo de los recursos para este tipo de estudios, lo que reduce la validez interna y externa de los resultados; se incluyeron pacientes con SCA con y sin elevación del ST en una pequeña población del noreste de México.

\section{Conclusión}

En pacientes con un SCA se demostró una relación entre polimorfismos relacionados con hemostasia e inflamación con ECMA, por lo que podrían considerarse marcadores de 
enfermedad coronaria. Se requiere una muestra mayor para confirmar estos resultados.

\section{Financiación}

El estudio fue aprobado y recibió financiamiento por el Fondo Sectorial de Investigación en Salud y Seguridad Social en la convocatoria SSA/IMSS/ISSTE-CONACYT 2002 (SALUD2002-CO1-7162).

\section{Conflicto de intereses}

Los autores declaran no tener ningún conflicto de intereses.

\section{Bibliografía}

1. Libby P, Theroux P. Pathophysiology of coronary artery disease. Circulation. 2005;111:3481-8.

2. Jerjes-Sánchez C, Comparan A, Canseco-Ávila LM, et al. Nuevos y futuros marcadores en la estratificación de los síndromes coronarios agudos. Arch Cardiol Mex. 2006;76:241-8.

3. Jerjes-Sánchez C, Comparán NA, Ibarra FM, et al. Marcadores hemostáticos y de inflamación en síndromes coronarios agudos y su asociación con eventos cardiovasculares adversos. Arch Cardiol Mex. 2006;76:366-75.

4. Canseco-Ávila LM, Jerjes-Sánchez C, Ortiz-López R, et al. Fibrinógeno. ¿Factor o indicador de riesgo cardiovascular? Arch Cardiol Mex. 2006;76:158-72.

5. Sambrook J, Fritsch EF, Maniatis T. Molecular cloning: a laboratory manual. $3^{\text {rd }}$ ed. Tomo III New York: Cold Spring Harbor Laboratory Press; 2001.

6. Bladbjerg EM, Gram J, Jespersen J, et al. Internal quality control of PCR-based genotyping methods: practical experiences. Vascul Pharmacol. 2002;39:127-9.

7. Comparan A, Palacios JM, Jerjes-Sánchez C. Leucocitos y su asociación con eventos cardiovasculares adversos en infarto con elevación del ST llevados a intervención coronaria percutánea. Arch Cardiol Mex. 2005;75:591-8.

8. Izaguirre AR, Zaldivar AH. Fibrinógeno como factor de riesgo. Arch Cardiol Mex. 2003;73:7-10.

9. Velázquez MO, Barinagarrementería AFS, Rubio GFA, et al. Morbilidad y mortalidad de la enfermedad isquémica del corazón y cerebrovascular en México. Arch Cardiol Mex. 2007;77:31-9.

10. Rosas M, Lara EA, Pastelín G, et al. Re-encuesta Nacional de Hipertensión Arterial (RENATA): consolidación mexicana de los factores de riesgo cardiovascular, cohorte nacional de seguimiento. Arch Cardiol Mex. 2005;75:96-111.

11. García Castillo A, Jerjes-Sánchez C, Martínez BP, et al. Registro Nacional de Síndromes Coronarios Agudos (RENASICA II). Arch Cardiol Mex. 2005;75:S18-30.

12. Rosas-Peral M, Attie F. Enfermedad cardiovascular. Primera causa de muerte en adultos de México y el mundo. Arch Cardiol Mex. 2007;77:91-3.

13. Lerman A, Zeiher AM. Endothelial function. Circulation. 2005;111:363-8.

14. Lupi Herrera E, Chuquiure Valenzuela E, Gaspar J, et al. De la placa vulnerable solitaria a la coronariopatía de múltiples vasos. De sus fundamentos a las implicaciones terapéuticas modernas. Una realidad clínica en el espectro de los SICA. Arch Cardiol Mex. 2006;76:6-34.

15. Mauriello A, Sangiorgi G, Fratoni S, et al. Diffuse and active inflammation occurs in both vulnerable and stable plaques of the entire coronary tree: a histopathology study of patients dying of acute myocardial infarction. J Am Coll Cardiol. 2005;45:1585-93.
16. Jerjes-Sanchez $C$. Venous and arterial thrombosis. A continuum spectrum of the same disease? Eur Heart J. 2005;26:1-2.

17. Liuzzo G, Santamaria M, Biasucci LM, et al. Persistent activation of nuclear factor Kappa-B signaling pathway in patients with unstable angina and elevated levels of C-reactive protein. J Am Coll Cardiol. 2007;49:185-94.

18. Donmez Y, Kanadasi M, Tanriverdi K, et al. Prothrombin 20210GA and factor $\mathrm{V}$ Leiden mutations in patients less than 55 years old with myocardial infarction. Jpn Heart J. 2004;3:505-12.

19. Franco RF, Santos SE, Elion J, et al. Prevalence of the G20210A polymorphism in the 3'-untranslated region of the prothrombin gene in different human populations. Acta Haematol. 1998;100:9-12.

20. Chan WP, Lee CK, Kwong YL, et al. A novel mutation of arg306 of factor V gene in Hong Kong Chinese. Blood. 1998;91:1135-9.

21. Gregg JP, Yamane AJ, Grody WW. Prevalence of the factor VLeiden mutation in four distinct American ethnic populations. Am J Med Genet. 1997;73:334-6.

22. Heinrich J, Ballseisen $L$, Schulte $H$, et al. Fibrinogen and factor $\mathrm{VII}$ in the prediction of coronary risk. Results from the PROCAM study in healthy men. Arterioscler Thromb. 1994;14:54-9.

23. Stec JJ, Silbershatz H, Tofler GH, et al. Association of fibrinogen with cardiovascular risk factors and cardiovascular disease in the Framingham Offspring Population. Circulation. 2000;102:1634-8.

24. Benderly M, Graff E, Reicher-Reiss H, et al., for the Bezafibrate Infarction Prevention (BIP) Study Group. Fibrinogen is a predictor of mortality in coronary heart disease patients. Arterioescler Thromb Vasc Biol. 1996;16:351-6.

25. De Maat $M$, Kastelein J, Jukema J, et al., on behalf of the REGRESS Group. -455G/A polymorphism of the $\beta$-fibrinogen gene is associated with the progression of coronary atherosclerosis in symptomatic men proposed role for an acute - phase reaction pattern of fibrinogen. Arterioscler Thromb Vasc Biol. 1998;8:265-71.

26. Lam KS, Ma OC, Wat NM, et al. Fibrinogen gene G/A-455 polymorphism in relation to fibrinogen concentrations and ischaemic heart disease in Chinese patients with type II diabetes. Diabetologia. 1999;42:1250-3.

27. Carter $A M$, Mansfield MW, Stickland $M H$, et al. Beta-fibrinogen gene $-455 \mathrm{G} / \mathrm{A}$ polymorphism and fibrinogen levels. Risk factors for coronary artery disease in subjects with NIDDM. Diabetes Care. 1996;19:1265-8.

28. Tybjaerg-Hansen A, Agerholm-Larsen B, Humphries SE, et al. A common mutation ( $\mathrm{G}-455-->A$ ) in the beta-fibrinogen promoter is an independent predictor of plasma fibrinogen, but not of ischemic heart disease. A study of 9.127 individuals based on the Copenhagen City Heart Study. J Clin Invest. 1997;99:3034-9.

29. Doggen CJ, Bertina RM, Cats VM, et al. Fibrinogen polymorphisms are not associated with the risk of myocardial infarction. Br J Haematol. 2000;110:935-8.

30. Laffan MA. Fibrinogen polymorphisms and disease. Eur Heart J. 2001;22:2224-6.

31. Yano K, Grove JS, Chen R, et al. Plasma fibrinogen as a predictor of total and cause-specific mortality in elderly Japanese-American men. Arterioscler Thromb Vasc Biol. 2001;21:1065-70.

32. Schmidt H, Schmidt R, Niederkorn K. Beta-fibrinogen gene polymorphism (C148T) is associated with carotid atherosclerosis: results of the Austrian Stroke Prevention Study. Arterioscler Thromb Vasc Biol. 1998;18:487-92.

33. Van Hooft FM, von Bahr SJ, Silveira A. Two common, functional polymorphisms in the promoter region of the beta-fibrinogen gene contribute to regulation of plasma fibrinogen concentration. Arterioscler Thromb Vasc Biol. 1999;19:3063-70.

34. Verschuur $M$, de Jong $M$, Felida $L$, et al. A hepatocyte nuclear factor-3 site in the fibrinogen beta promoter is important for interleukin 6-induced expression, and its activity is influenced 
by the adjacent $-148 \mathrm{C} / \mathrm{T}$ polymorphism. J Biol Chem. 2005;280:16763-71.

35. lacoviello L, Vischetti M, Zito F, et al. Genes encoding fibrinogen and cardiovascular risk. Hypertension. 2001;38:1199-203.

36. Anderson GM, Shaw AR, Shafer JA. Functional characterization of promoter elements involved in regulation of human $B$ betafibrinogen expression. Evidence for binding of novel activator and repressor proteins. J Biol Chem. 1993;268:22650-5.

37. Dalmon J, Laurent $M$, Courtois $G$. The human beta fibrinogen promoter contains a hepatocyte nuclear factor 1dependent interleukin-6-responsive element. Mol Cell Biol. 1993;13:1183-93.

38. Gervois P, Vu-Dac N, Kleemann R, et al. Negative regulation of human fibrinogen gene expression by peroxisome proliferator-activated receptor alpha agonists via inhibition of CCAAT box/enhancer-binding protein beta. J Biol Chem. 2001;276:33471-7.

39. Andrioli G, Minuz P, Solero P, et al. Defective platelet response to arachidonic acid and thromboxane $\mathrm{A} 2$ in subjects with $\mathrm{Pl}$ (A2) polymorphism of beta (3) subunit (glycoprotein IIIa). $\mathrm{Br}$ J Haematol. 2000;110:911-8.

40. Doggen CJM, Cats VM, Bertina RM, et al. Interaction of coagulation defects and cardiovascular risk factors: increased risk of myocardial infarction associated with factor $V$ Leiden or prothrombin 20210A. Circulation. 1998;97:1037-44.

41. Redondo M, Watzke HH, Stucki B, et al. Coagulation factors II, V, VII, and X, prothrombin gene 20210G--> A transition, and factor $\mathrm{V}$ Leiden in coronary artery disease: high factor $\mathrm{V}$ clotting activity is an independent risk factor for myocardial infarction. Arterioscler Thromb Vasc Biol. 1999;19:1020-5.

42. Kohler HP, Stickland MH, Ossei-Gerning N, et al. Association of a common polymorphism in the factor XIII gene with myocardial infarction. Thromb Haemost. 1998;79:8-13.

43. Wagner DD. New links between inflammation and thrombosis. Arterioscler Thromb Vasc Biol. 2005;25:1321-4.

44. Lacoviello L, di Castelnuovo A, de Knijff de P, et al. Alu-repeat polymorphism in the tissue-type plasminogen activator gene,
t-PA levels and risk of familial myocardial infarction. Fibrinolysis. 1996;10 suppl 2:13-6.

45. Van der Boom JG, de Knijff $P$, Haverkate F, et al. Tissue plasminogen activator and risk of myocardial infarction: the Rotterdam study. Circulation. 1997;95:2623-7.

46. Ridker PM, Baker MT, Hennekens $\mathrm{CH}$, et al. Alu-repeat polymorphism in the gene coding for tissue-type plasminogen activator (t-PA) and risks of myocardial infarction among middle-aged men. Arterioscler Thromb Vasc Biol. 1997; 17:1687-90.

47. Makalowski W, Mitchell GA, Labuda D. Alu sequences in the coding regions of mRNA: a source of protein variability. Trends Genet. 1994;10:188-93.

48. Chandler WL, Levy WC, Stratton JR. The circulatory regulation of TPA and UPA secretion, clearance, and inhibition during exercise and during the infusion of isoproterenol and phenylephrine. Circulation. 1995;92:2984-94.

49. Emeis JJ. Local fibrinolysis. J Thromb Haemost. 2005;3:1945-6.

50. Brommer EJ. The level of extrinsic plasminogen activator (t-PA) during clotting as a determinant of the rate of fibrinolysis, inefficiency of activators added afterwards. Thromb Res. 1984;34:109-15.

51. Fox KA, Robison AK, Knabb RM, et al. Prevention of coronary thrombosis with subthrombolytic doses of tissue-type plasminogen activator. Circulation. 1985;72:1346-54.

52. Jern C, Ladenvall P, Wall U. Gene polymorphism of t-PA is associated with forearm vascular release rate of t-PA. Arterioscler Thromb Vasc Biol. 1999;19:454-9.

53. Sartori MT, Saggiorato G, Spiezia L, et al. Influence of the Alurepeat I/D polymorphism in t-PA gene intron 8 on the stimulated t-PA release after venous occlusion. Clin Appl Thromb Hemost. 2003;9:63-9.

54. Mahmud E, Cavendish JJ, Tsimikas S, et al. Elevated plasma fibrinogen level predicts suboptimal response to therapy with both single - and double - bolus eptifibatide during percutaneous coronary intervention. J Am Coll Cardiol. 2007;49:2163-71. 